The authors analyzed specimens from 73 patients with advanced NSCLC treated with gefitinib or erlotinib. EGFR mutations were identified in $9.8 \%$ of the patients and were significantly associated with objective response to TKI therapy $(P<0.0001)$, but not with overall survival $(P=0.08)$. Increased EGFR copy numbers were detected in $54.2 \%$ of patients; however, copy-number status did not correlate with favorable response to EGFR-TKIs $(P=0.48)$ or overall survival $(P=0.68)$. KRAS mutations were detected in $22.8 \%$ of patients and were significantly associated with lack of response to TKI therapy $(P=0.04)$ and shorter median time to progression $(P=0.0025)$, but not with overall survival $(P=0.62)$. The probability of having the highest response rate was greater than $99.7 \%$ in patients with both EGFR mutation and increased EGFR copy number. Patients with $K R A S$ mutation with or without increased EGFR copy number were at the highest risk of progressive disease, with the probability of having the highest progressive-disease rate calculated as greater than $96.5 \%$.

The authors conclude that KRAS mutation is a significant predictor of poor prognosis and poor response to EGFR-TKIs in patients with NSCLC.

Original article Massarelli E et al. (2007) KRAS mutation is an important predictor of resistance to therapy with epidermal growth factor receptor tyrosine kinase inhibitors in non-small-cell lung cancer. Clin Cancer Res 13:2890-2896

\section{Gemcitabine plus capecitabine is effective in advanced pancreatic cancer}

For patients with advanced or metastatic pancreatic cancer, gemcitabine is the standard therapy; however, although gemcitabine therapy increases overall survival, response rates are low. Herrmann et al. compared the safety and efficacy of gemcitabine plus capecitabine (GemCap) with that of gemcitabine monotherapy in patients with pancreatic cancer.

This phase III trial included 319 patients with primary inoperable or metastatic pancreatic cancer who were randomized to receive GemCap or gemcitabine alone. Patients receiving the combination regimen had a median overall survival of 8.4 months compared with 7.2 months for those on single therapy $(P=0.234)$. Patients with a good Karnofsky performance score
(90-100) who received GemCap had a significantly prolonged median overall survival compared with patients on gemcitabine alone (10.1 months vs 7.4 months). This prolonged survival translated to an average survival gain of $36 \%$ for patients in the GemCap arm compared with the gemcitabine only arm $(P=0.014)$. Both groups had similar overall frequencies of grade 3 or 4 hematologic adverse events, with neutropenia being the most common event. Grade 3 or 4 nonhematologic adverse events were uncommon.

The authors conclude that the GemCap regimen is a safe alternative to single agent gemcitabine therapy in patients with advanced or metastatic pancreatic cancer who have a good performance status.

Original article Herrmann R et al. (2007) Gemcitabine plus capecitabine compared with gemcitabine alone in advanced pancreatic cancer: a randomized, multicenter, phase III trial of the Swiss Group for Clinical Cancer Research and the Central European Cooperative Oncology Group. J Clin Oncol 25: $2212-2217$

\section{Improved outcome in patients with soft tissue sarcoma treated at high-volume centers}

Soft tissue sarcomas (STSs) are a rare and heterogeneous group of neoplasms. The low incidence of these sarcomas means that many surgical centers have little experience in treating the disease. To determine the effect of surgical center case volume on outcome for STS, Gutierrez et al. conducted a large populationbased study using data from the Florida state cancer registry.

During the period 1981-2001, 256 centers in Florida performed at least one resection for STS. The researchers defined high-volume centers (HVCs) as those above the $67^{\text {th }}$ percentile of procedures when all 256 facilities were ranked by operative volume. According to this definition, 7 institutions were classified as HVCs, performing 1,504 of the 4,673 procedures conducted during the study period. Both 30-day and 90-day mortality were lower at HVCs than at the other (low-volume) centers (0.7\% vs $1.5 \%, P=0.028$, and $1.6 \%$ vs $3.6 \%$, $P=0.001$, respectively). In addition, the median survival of patients treated at HVCs was significantly longer than that of patients treated at low-volume centers (40 months vs 37 months; $P=0.002)$. The superior median survival at 